\title{
Innovación y empresas: un análisis de sus características a nivel regional
}

\author{
Innovation and companies: an analysis of its characteristics on a regional level \\ Carmen Araneda-Guirriman ${ }^{1 *}$, Liliana Pedraja-Rejas ${ }^{1}$, Emilio Rodríguez Ponce ${ }^{2}$
}

\begin{abstract}
RESUMEN
La presente investigación tiene por finalidad describir a nivel de región la relación que tiene la cantidad de empresas que introducen nuevas innovaciones al mercado en las distintas Regiones de Chile y el volumen de empresas que en esos territorios capacitan en innovación y aquellas que poseen infraestructura que estas poseen para el desarrollo de las actividades de Investigación y Desarrollo. Para este fin se trabaja con los resultados de la Octava Encuesta de Innovación en Empresas que da cuenta del periodo 2011-2012 realizada por el Ministerio de Economía, Fomento y Turismo de Chile. La información cuantitativa fue procesada por medio del análisis estadístico descriptivo y análisis de regresión múltiple. Los resultados indican que a nivel de regiones existe una relación entre la cantidad de empresas que realizan capacitaciones en innovación y el volumen de compañías que poseen la estructura para la investigación y desarrollo, y la cantidad de empresas que introducen innovaciones al mercado las empresas, siendo un punto inicial para el análisis de los elementos que se encuentran asociados a la innovación en las diversas regiones de Chile.
\end{abstract}

Palabras clave: innovación, empresas, regiones, investigación y desarrollo.

\begin{abstract}
This research aims to describe the regional level, the relationship of the number of companies that introduce new innovations to market in different regions of Chile and the volume of business in those territories trained in innovation and those who own infrastructure they have for the development of $R \& D$ activities. To this end it was worked with the results of the Eighth Survey Innovation in Companies realize the 2011-2012 period by the Ministry of Economy, Development and Tourism of Chile. Quantitative information was processed using descriptive statistical analysis and multiple regression analysis. The results show that the level of regions there is a relationship between the number of companies conducting training in innovation and volume of companies that have the structure for research and development, and the number of companies introducing innovations to market companies, with a starting point for the analysis of the elements that are associated with innovation in the various regions of Chile.
\end{abstract}

Key words: innovation, business, regions, research and development.

\section{Introducción}

En la actualidad el conocimiento y la innovación pueden considerarse como dos aspectos inherentes al desarrollo de las sociedades actuales, ya que el crecimiento económico de una nación depende cada vez menos de la acumulación de los factores tradicionales, vinculándose cada vez más a las capacidades de generar y aplicar nuevos conocimientos, lo que ha derivado en la noción de las economías basadas en el conocimiento (Fernández de Lucio et al., 2011).

De igual modo, la digitalización de la información, junto con la utilización de internet, han facilitado un uso intensivo en la aplicación del conocimiento, transformándolo en un elemento predominante de la creación de valor, tanto para las personas como para las organizaciones y países (Rodríguez-Ponce, 2009). La generación de nuevo conocimiento y el progreso tecnológico son, por tanto, parte de la realidad actual y a su vez se configuran como aspectos determinantes del crecimiento económico, situación que se encuentra estrechamente vinculada al territorio, razón por la que existe un interés por potenciar el desarrollo económico desde la esfera regional (Listerri y Pietrobelli, 2011). En este sentido, los resultados de un estudio realizado por Pedraja-Rejas et al. (2009) en empresas del norte

\footnotetext{
Centro de Estudios Ceuta, Universidad de Tarapacá.

2 Instituto de Alta Investigación, Universidad de Tarapacá.

* Autor para correspondencia: caraneda@uta.cl
}

Fecha de Recepción: 6 Septiembre, 2015.

Fecha de Aceptación: 18 Octubre, 2015. 
de Chile muestran que en las empresas privadas el crear y compartir conocimiento son variables esenciales que impactan sobre la eficacia, junto con la aplicación de conocimiento, la que tiene un efecto más bien moderado.

Por tanto, el objetivo del análisis que se muestra en el presente trabajo es explorar en las regiones de Chile la relación que tiene el volumen de empresas que realizan innovación con la cantidad de empresas que poseen infraestructura para la investigación y también aquellas que capacitan en innovación a su personal, pues se considera que aquellas regiones que poseen más empresas con estas dos últimas características son aquellas que logran tener una mayor cantidad de empresas que introducen innovaciones al mercado.

En este sentido, la innovación se puede considerar como un proceso amplio arraigado en las capacidades de generación, transmisión y asimilación de conocimiento, la que es generada mediante interacciones entre organizaciones e individuos en diversos niveles (OCDE, 2009). Por tanto, se debe hacer referencia a los sistemas de innovación, cuya finalidad es analizar las transiciones de sustentabilidad y otorgar las herramientas para detectar las debilidades del sistema (Jacobsson y Bergek, 2011). En consecuencia, resulta relevante considerar los atributos sistémicos del proceso de innovación por medio del sistema de innovación (Radosevic y Kriaucioniene, 2014).

En este contexto regional, enfatizado en esta investigación, cobra especial importancia hablar de los sistemas de innovación regional (Cooke, 2008), el que es entendido como un sistema de redes de innovación e instituciones localizadas en un área geográfica, con interacciones firmes y regulares que incentivan la innovación en la región (Kostiainen, 2002). Es así como un sistema de innovación es el escenario para que los procesos de innovación puedan ser descritos en términos de recursos y flujos de conocimientos conducen a avances tecnológicos (Padmore et al., 1998). De igual modo, un sistema regional de innovación hace referencia a un sistema de innovación ubicado en una región, un nivel por debajo del sistema nacional de innovación que podría tener homogeneidad cultural o histórico, donde el desarrollo económico localizado puede ser (Cooke, 2001). Es necesario precisar que un sistema regional de innovación difiere en su nivel de desarrollo debido a su capacidad para capitalizar nuevos recursos de conocimientos y sus capacidades productivas (Tiffin y Kunc, 2011). En efecto, un sistema de innovación está constituido por componentes, que son las partes operativas del sistema, como organizaciones, empresas, bancos, relaciones entre estos y sus características (Carlsson et al., 2002).

Del mismo modo se debe tener en consideración que el conocimiento en la industria cambia velozmente, esto implica que las empresas requieran crear nuevo conocimiento para avanzar del mismo modo (Pedraja Rejas et al., 2006). Es así como los resultados de un estudio realizado con compañías nórdicas evidencian que las pequeñas y medianas empresas pueden crear innovaciones con diferentes combinaciones de recursos. La combinación más común es el patrimonio, Investigación y Desarrollo (I+D), cooperación, redes, industria del conocimiento y reputación. A excepción del capital financiero en forma de patrimonio, que es condición necesaria para la innovación en las empresas, las necesidades de recurso varían entre innovaciones tecnológicas y modelos de negocios (Halme y Korpela, 2014). En relación con lo señalado anteriormente se encuentran los resultados de una investigación efectuada por Leiponen (2012), que indican que la inversión en Investigación y Desarrollo juega un rol económica y estadísticamente significativo al momento de abordar la innovación de servicios, lo que está directamente relacionado con la creciente participación de las empresas de servicios en actividades de I+D. Esto se relaciona con el hecho de que los nuevos productos y servicios se han desarrollado tradicionalmente en los procesos de I+D interna (Schneckenberg, 2015).

En este marco contextual de innovación, el incremento y la calificación de recurso humano en las actividades de I+D son elementos esenciales para el desarrollo del sistema de ciencia y tecnología, ello implica a su vez un incremento del capital intelectual (Silva et al., 2013). Asimismo, los resultados de un estudio de D'Agostino et al. (2013) indican que para las empresas multinacionales la inversión en I+D en los países mejora la eficacia de la producción de conocimiento de la región de origen. Por tanto, las políticas de promoción de la I+D por parte del gobierno podrían conducir al desarrollo de tecnologías endógenas (Mah, 2015). Por lo demás, la capacitación es la forma de lograr la transferencia de conocimiento entre el personal de una empresa (Zhao et al., 2014), esto es fundamental para los procesos de innovación; la importancia de la vinculación entre innovación y la gestión de recursos humanos ha sido abordada por Laursen y 
Foss (2003). De igual modo, según los resultados de un estudio llevado a cabo en Turquía, demuestran que gran parte de los gestores de I+D consideran de vital importancia el crecimiento y la formación del personal, ya sea al interior de la empresa o por vía externa, conformando bibliotecas digitales y animando a los empleados a estudiar un doctorado o una maestría, mientras se internaliza una cultura corporativa orientada hacia la innovación (Duygulu et al., 2015).

Es oportuno mencionar que el mayor valor agregado de una organización está conformado por su personal, por tanto, un componente humano capacitado puede llevar a cabo las tareas de mejor forma, es decir, una empresa existe en relación con la formación del personal que la conforma, ya que el concepto de capital humano representa el valor del total de empleados incluyendo todos y cada uno de sus niveles en la organización (Correa et al., 2015). Complementariamente se puede establecer que, de acuerdo con los resultados de un estudio realizado a la innovación en las regiones de Chile, el número de empresas que realizan innovaciones se ve determinado, en primera instancia, por el porcentaje de gasto en investigación y desarrollo que es ejecutado por las instituciones de educación superior, por tanto las universidades juegan un rol trascendente, no solo en el desarrollo de capital humano, sino que también en la producción de conocimiento que al momento de ser transferidos al medio van generando sinergias al interior de las regiones (Araneda et al., 2015). Asimismo, vale destacar que el crecimiento económico de las regiones europeas está significativamente relacionado con su conocimiento y la dotación de su capital humano (Hajek et al., 2014).

\section{Metodología}

La metodología de la investigación es cuantitativa, teniendo un propósito exploratorio y descriptivo al pretender describir cómo la cantidad de empresas que efectúan capacitación en innovación y el volumen de empresas que cuenta con una infraestructura $\mathrm{I}+\mathrm{D}$ en las regiones influyen en la cantidad de empresas que lanzan innovaciones al mercado en dichos territorios. Es valioso señalar que para este estudio se utilizaron fuentes de datos secundarios, obtenidos de la Octava Encuesta de Innovación en Empresas que da cuenta del periodo 2011-2012, instrumento que sigue el modelo y lineamientos generales establecidos por la OCDE y la Community Innovation Survey (CIS), efectuada por el Ministerio de Economía, Fomento y Turismo de Chile y que fue aplicada a diversas empresas. Es sustancial mencionar que esta encuesta mide variables tales como el tipo de innovación, grado de novedad, actividades innovativas, entre otros, que realizan las empresas chilenas en las distintas regiones de Chile, permitiendo información cuantitativa y cualitativa (Ministerio de Economía, Fomento y Turismo, 2014).

A partir de los resultados cuantitativos de dicha encuesta, se utilizaron las siguientes variables (Tabla 1).

El procesamiento más pertinente para los datos es el estadístico, mediante un análisis estadístico descriptivo, para visualizar cómo se comportaban las variables. Posterior a ello, y con el fin de visualizar si efectivamente existe una relación entre las variables del estudio, según la ecuación que se señala a continuación, se realizó un análisis de correlaciones, con el fin de tener una aproximación bivariada al fenómeno, para finalmente efectuar una regresión lineal múltiple con la siguiente ecuación de regresión:

$$
\mathrm{Vx}=\beta_{0}+\mathrm{a} \beta_{1}+\mathrm{b} \beta_{2}+\varepsilon_{\mathrm{i}}
$$

Donde $\mathrm{V}_{\mathrm{x}}$ es la variable dependiente, es decir, las empresas que introducen innovaciones al

Tabla 1. Variables y medidas.

\begin{tabular}{lll}
\hline Variable & Indicador & Fuente de información \\
\hline Empresas que realizaron capacitación en & $\begin{array}{l}\text { Número de empresas que realizan } \\
\text { capacitación en innovación }\end{array}$ & $\begin{array}{l}\text { Resultados de la Octava encuesta de } \\
\text { innovación }\end{array}$ \\
$\begin{array}{l}\text { Número de empresas que poseen unidad en empresas, 2011-2012 } \\
\text { Empresas con estructura I+D }\end{array}$ & \\
& $\begin{array}{l}\text { formal, departamento o laboratorio I+D } \\
\text { según región }\end{array}$ \\
Empresas que realizan introducción de & $\begin{array}{l}\text { Número de empresas que realizan } \\
\text { introducción de innovaciones al mercado }\end{array}$ \\
\hline
\end{tabular}

Fuente: Elaboración propia. 
mercado; $\beta_{0}$ constante del modelo; a es la variable independiente Empresas con estructura I+D; $\beta_{1}$ Factor de ponderación; b variable independiente Empresas que realizaron capacitación en innovación; $\beta_{2}$ Factor de ponderación; y $\varepsilon_{\mathrm{i}}$ : error residual del modelo.

\section{Resultados y Discusión}

En primera instancia, al analizar los resultados descriptivos, podemos observar que en promedio el número de empresas que introducen innovaciones al mercado es en promedio de 238 a nivel nacional. Es factible mencionar que el valor máximo de empresas corresponde a la región Metropolitana, y el valor mínimo a la región de Aysén. En relación con las empresas que poseen Unidad I+D se encuentra que en promedio 155 con una variación de 362,9 alrededor de la media, lo que evidencia la presencia de valores extremos en la distribución. Por otra parte, en promedio 425 empresas capacitan a su personal para la innovación. La Región Metropolitana es la que concentra la mayor cantidad de empresas que introducen innovaciones al mercado, que capacitan a su personal para la innovación y que poseen unidades dedicadas para las acciones de investigación y desarrollo.

De acuerdo con los resultados de la Tabla 2, se puede apreciar que existe una concentración del número de empresas que introducen innovaciones al mercado, esto podría explicarse debido a que las ciudades más innovadoras también tienen altas concentraciones de instituciones como las universidades que realizan investigación y desarrollo, junto con las empresas de los sectores industriales (Simmie, 2002), como sucede por ejemplo en el caso de la Región Metropolitana, no obstante eso se debiera profundizar en un siguiente estudio.

A partir de los resultados de la Tabla 3 se puede establecer que el número de empresas que introducen innovaciones al mercado por región se ve directamente influenciado por la cantidad de empresas que poseen estructura I+D y que además invierten en capacitación para la innovación de su personal en dichos territorios ( $\mathrm{p}<0,001 ; \mathrm{R} 0,988)$. Con relación a la importancia que tiene la capacitación en innovación que realiza la empresa, esta resulta

Tabla 2. Estadísticas descriptivas.

\begin{tabular}{llcccc}
\hline & $\mathrm{N}$ & Mínimo & Máximo & Media & Desv. típ. \\
\hline Número de empresas que introducen innovaciones al mercado & 15 & 9 & 2048 & 238.13 & 511.245 \\
Número de empresas que posee unidad/depto/lab I+D & 15 & 1 & 1398 & 155.07 & 362.975 \\
Número de empresas que capacitan para la innovación & 15 & 9 & 3237 & 425.13 & 790.179 \\
N válido (según lista) & 15 & & & & \\
\hline
\end{tabular}

Fuente: Procesamiento información Spss.

Tabla 3. Resumen del modelo de regresión.

\begin{tabular}{lc}
\hline & $\begin{array}{c}\text { Número de empresas que realizan innovación } \\
\text { de proceso y de producto por región }\end{array}$ \\
\hline $\mathrm{R}$ & 0,988 \\
$\mathrm{R}$ cuadrado & 0,976 \\
$\mathrm{R}$ cuadrado corregida & 0,972 \\
Error típ. de la estimación & 85,327 \\
$\mathrm{~F}$ & 245,295 \\
Sig. & $0,000 \mathrm{a}$ \\
Test t constante & $-0,423$ \\
Sig. Test T constante & 0,680 \\
Test t N ${ }^{\circ}$ de empresas que posee unidad / depto. / laboratorio de I+D & 2,823 \\
Sig. Test t N ${ }^{\circ}$ de empresas que posee unidad / depto. / laboratorio de I+D & 0,015 \\
Coeficiente beta de empresas que posee unidad / depto. / laboratorio de I+D & 0,477 \\
Test t Número de empresas que capacitan para la innovación & 3,485 \\
Sig. Test Número de empresas que capacitan para la innovación & 0,005 \\
Coeficiente beta Número de empresas que capacitan para la innovación & 0,551 \\
\hline
\end{tabular}

a. Variables predictoras: (Constante), Número de empresas que introducen innovaciones al mercado. Fuente: Procesamiento información Spss. 
fundamental, ya que el capital humano es un factor de elevada relevancia al momento de abordar los procesos de innovación, estos enfatizan la importancia que tiene la educación formal y de capacitación en instituciones externas (Bassan et al., 2013).

En este orden, los resultados de un estudio realizado por Smith et al. (2012) identificó una relación, aunque débil, entre la capacitación y la innovación al interior de las empresas. Por el contrario, otro estudio confirma que la capacitación del capital humano de una empresa agrícola australiana y el uso de innovaciones tienen un impacto significativamente beneficioso en el incremento del rendimiento de los cultivos alcanzados (Xayavong et al., 2015). Por tanto, se puede establecer que la capacitación se configura como un elemento diferenciador entre las compañías el considerar las funciones de investigación como una actividad permanente y no de forma esporádica (Czarnitzki et al., 2011). La capacitación se focaliza en la mejora del rendimiento actual o futuro de una persona en su puesto o lugar de trabajo actual (Correa et al., 2015). En consecuencia, en las regiones analizadas, la cantidad de empresas que capacitan para la innovación a sus empleados se relaciona con el número de empresas que introducen innovaciones al mercado en dichos territorios.

En efecto, hoy se puede considerar como una gran preocupación la ausencia en las pequeñas y medianas empresas de un departamento formal de investigación y desarrollo, considerando además que la preparación de los individuos, en los diferentes niveles de la estructura organizacional, es fundamental para orientar el trabajo hacia la innovación (Bassan et al., 2013). Es así como la capacitación en innovación que realizan las empresas también tiene un impacto en la introducción de innovaciones al mercado.

Es propicio destacar que ambas variables, tanto el número de empresas que posee la infraestructura para la $\mathrm{I}+\mathrm{D}$ como aquellas que capacitan en innovación que realizan las empresas, tienen un impacto directamente proporcional con el volumen de compañías que introduce innovaciones al mercado, configurándose ambos elementos como factores a considerar al momento de analizar la innovación que se lleva a cabo a lo largo de las regiones de Chile. A este respecto, se precisa que en las últimas décadas Chile ha tenido un desempeño menos que regular en lo que respecta a innovación, a pesar de los indicadores económicos, destinando una parte muy pequeña del PIB en investigación y desarrollo
(Cruz, 2008). En efecto, para el 2012 el gasto en I+D, como porcentaje del PIB, fue de $0,35 \%$ para Chile, muy por debajo al 2,4\% de la OCDE (Ministerio de Economía, Fomento y Turismo, 2014).

\section{Conclusiones}

La innovación en las empresas se configura como un elemento capaz de generar ventaja competitiva en los territorios, situación que cobra mayor relevancia al momento de analizar las empresas a nivel de las regiones de Chile. Con relación a este análisis exploratorio, se puede establecer que existen factores que tienen un impacto sobre el número de empresas que introducen innovaciones al mercado. En este sentido, resulta central considerar el número de compañías que a nivel regional tiene infraestructura de investigación y desarrollo que poseen las empresas y por otra parte aquellas que efectúan capacitaciones en torno a la innovación para sus trabajadores. Los resultados también muestran la concentración territorial que se da en estos factores en la Región Metropolitana, lo que genera gran diferencia respecto de las otras regiones del país, elemento que se debe considerar al momento de definir políticas o programas de incentivo a la innovación.

Resulta de crucial importancia dentro de una empresa al momento de abordar el tema de la innovación, el contar con la infraestructura y con las capacidades en su capital humano. Las regiones con mayor concentración de empresas que poseen unidad $\mathrm{I}+\mathrm{D}$ y que capacitan a su personal en innovación son aquellas regiones que logran introducir más innovaciones al mercado. En efecto, cuando una empresa adquiere conocimiento mediante la capacitación se difunde conocimiento adquirido y acumulado para convertirse en un activo valioso por medio de un mecanismo de integración del conocimiento, mejorará el rendimiento de la formación (Zhao et al., 2014).

Vale mencionar que al ser un estudio exploratorio, se debe continuar profundizando en otros factores asociados a la innovación en las empresas como es la transferencia de conocimientos de otros organismos dedicados a las actividades I+D, así como también la vinculación con la universidad, entre otros.

\section{Limitaciones}

Es primordial precisar que este es un estudio exploratorio que entrega una aproximación general, 
que aborda y analiza solo dos factores asociados a la innovación de empresas, y a nivel regional, que sirve para una posterior profundización de este fenómeno en las regiones, haciendo otras bajadas de análisis más específicas de las propias empresas y otras organizaciones y su relación con estas.

\section{Agradecimientos}

Este trabajo fue posible gracias al patrocinio de la Universidad de Tarapacá por medio del proyecto UTA Mayor 2483 y al apoyo del Centro de estudios CEUTA.

\section{Literatura Citada}

Araneda-Guirriman, C.; Pedraja-Rejas, L.; Rodríguez Ponce, E. 2015. Innovación en las regiones de Chile: una aproximación desde el análisis de sus empresas. Idesia (Arica), 33(1): 125-133.

Bassan, C.R.B.; Abreu, A.; Woida, L.M.

2012. Innovation management through knowledge and organizational socialization; Gestión de la innovación a través del conocimiento y la socialización de organización. Informação \& Informação, 17(2): 103-132.

Carlsson, B.; Jacobsson, S.; Holmén, M.; Rickne, A.

2002. Innovation systems: analytical and methodological issues. Research policy. 31(2): 233-245.

Cooke, $\mathrm{P}$.

2008. Regional Innovation Systems, Clean Technology \& Jacobian Cluster-Platform Policies. Regional Science Policy \& Practice, 1(1): 23-45.

Cooke, $\mathrm{P}$.

2001. Regional innovation systems, clusters, and the knowledge economy. Industrial and corporate change, 10(4): 945-974.

Correa, J.E.L.; Camacho, M.J.A.; Mosqueda, R.E.

2015. Application of a Model of Human Capital to Promote the Competitiveness of Small and Medium Enterprises. Review of Business \& Finance Studies, 6(3), 31-44.

Chen, J-H.

2015. The Trading Costs of Early Earnings Release: The Case of Hewlett-Packard Company. Review of Business \& Finance Studies, 6(3): 1-10.

Cruz, A.

2008. La Ruta a la Innovación en Chile. Journal of Technology Management \& Innovation, 3(1), 1-9.

Czarnitzki, D.; Hanel, P.; Rosa, J.

2011. Evaluating the impact of R\&D tax credits on innovation: A microeconometric study on Canadian firms. Research Policy, 40(2): 217-229.

D’Agostino, L.M.; Laursen, K.; Santangelo, G.D.

2013. The impact of R\&D offshoring on the home knowledge production of OECD investing regions. Journal of Economic Geography, 13(1): 145-175.

Duygulu, E.; Ozeren, E.; Bagiran, D.; Appolloni, A.; Mavisu, M. 2015. Gaining insight into innovation culture within the context of R\&D centres in Turkey. Int. J. Entrepreneurship and Innovation Management, 19(1/2): 117.

Fernández de Lucio, I.; Vega Jurado, J.; Gutiérrez Gracia, A. 2011. Ciencia e innovación: una relación compleja y evolutiva. Arbor, 187: 1077-1089.

Hajek, P.; Henriques, R.; Hajkova, V.

2014. Visualising components of regional innovation systems using self-organizing maps-Evidence from European regions. Technological Forecasting and Social Change, 84: 197-214.
Halme, M.; Korpela, M.

2014. Responsible Innovation Toward Sustainable Development in Small and Medium-Sized Enterprises: a Resource Perspective.Business Strategy and the Environment, 23(8): 547-566.

Jacobsson, S.; Bergek, A.

2011. Innovation system analyses and sustainability transitions: Contributions and suggestions for research.Environmental Innovation and Societal Transitions, 1(1): 41-57.

Kostiainen, J.

2002. Urban economic policy in the network society. Helsinki, Tekniikan Akateemisten Liitto.

Laursen, K.; Foss, N.J.

2003. New human resource management practices, complementarities and the impact on innovation performance. Cambridge Journal of economics, 27(2): 243-263.

Leiponen, A.

2012. The benefits of R\&D and breadth in innovation strategies: a comparison of Finnish service and manufacturing firms. Industrial and Corporate Change, 21(5):1255-1281.

Llisterri, J.J.; Pietrobelli, C.

2011. Los Sistemas Regionales de Innovación en América Latina: conclusiones y recomendaciones. In: Llisterri, J. J.; Pietrobelli, C. Los sistemas regionales de innovación en América Latina. Banco Interamericano de Desarrollo, Washington, D.C. pp. 104-122.

Mah, J.S.

2015. R\&D policies and development of technology-intensive industries of Taiwan. Progress in Development Studies, 15(2): 125-138.

Ministerio de Economía, Fomento y Turismo.

2014. Encuesta Nacional de Investigación y Desarrollo e Innovación. Antecedentes Metodológicos.

OECD.

2009. Policy Responses to the Economic Crisis: Investing in Innovation for Long-Term Growth. OECD, Paris. 37 p.

Padmore, T.; Schuetze, H.; Gibson, H.

1998. Modeling systems of innovation: An enterprise-centered view. Research Policy, 26(6): 605-624.

Pedraja-Rejas, L.; Rodríguez-Ponce, E.; Rodríguez-Ponce, J. 2009. La influencia de la gestión del conocimiento sobre la eficacia organizacional: A study in public institutions and private firms. Revista Facultad de Ingeniería Universidad de Antioquia, 47: 218-227.

Pedraja-Rejas, L.; Rodríguez-Ponce, E.; Rodríguez-Ponce, J. 2006. Sociedad del conocimiento y dirección estratégica: Una propuesta integradora. Interciencia, 31(8): 570-576. 
Radosevic, S.; Kriaucioniene, M.

2014. Higher education in national innovation. In Bridges, D.; Juceviciene, P.; Jucevicius, R.; Mclaughlin, T. H.; Stankeviciute, J. (Eds.). Higher education and national development: Universities and societies in transition. Routledge. pp. 135-160.

Rodríguez-Ponce, E.

2009. El rol de las universidades en la sociedad del conocimiento y en la era de la globalización: Evidencia desde Chile. Interciencia, 34(11): 822-829.

Schneckenberg, D.

2015. Open innovation and knowledge networking in a multinational corporation. Journal of Business Strategy, 36(1): 14-24.

Silva, D.; Romero, F.; Vieira, F.D.

2013. Effects of Technological Innovation on Knowledge Acquisition Inside the Organization: A Case Study. In. Galbraith, B. (Ed.). Proceedings of the 9th European Conference on Innovation and Entrepreneurship. Academic Conferences and Publishing International (ACPI). Belfast, UK. Pp. 791-794.
Simmie, J.

2002. Knowledge spillovers and reasons for the concentration of innovative SMEs. Urban Studies, 39(5-6), 885-902.

Smith, A.; Courvisanos, J.; Tuck, J.; McEachern, S.

2012. Building the Capacity to Innovate: The Role of Human Capital-Support Document. National Centre for Vocational Education Research (NCVER), Adelaide, Australia. 41 p.

Tiffin, S.; Kunc, M.

2011. Measuring the roles universities play in regional innovation systems: a comparative study between Chilean and Canadian natural resource-based regions. Science and Public Policy, 38(1); 55-66.

Xayavong, V., Kingwell, R.; Islam, N.

2015. How training and innovation link to farm performance: a structural equation analysis. Australian Journal of Agricultural and Resource Economics, 59: 1-16.

Zhao, J.; Qi, Z.; De Pablos, P.O.

2014. Enhancing enterprise training performance: Perspectives from knowledge transfer and integration. Computers in Human Behavior, 30: 567-573. 
